\title{
Microearthquakes in and Near Long Valley, California
}

\author{
Don W. Steeples ${ }^{1}$ and A. M. Pitt \\ U.S. Geological Survey, Menlo Park. California 94025
}

\begin{abstract}
Sixteen portable seismograph stations were deployed in the vicinity of the Long Valley geothermal area, California, from April 27 to June 2, 1973. Only minor microearthquake activity was detected in the Long Valley caldera, but a high level of activity was detected to the south and east of the caldera. The abrupt spatial seismicity decrease at the southern boundary of the caldera suggests that the caldera is either structurally less competent than the surrounding crust or is at a junction of different regional tectonic deformation trends. No significant attenuation or delays occurred for either local $P$ or $S$ waves that traversed the caldera.
\end{abstract}

\section{INTRODUCTION}

Sixteen portable seismograph stations were deployed in the area of Long Valley, California, from April 27 to June 2, 1973, as part of a U.S. Geological Survey (USGS) multidisciplinary investigation for possible geothermal resources in the Long Valley caldera. The principal objective of this study was to detect and locate microearthquakes in the Long Valley caldera, an area of present-day hydrothermal activity.

Facca and Tonani [1964] suggested that the delineation of specific faults would be a valuable tool in the search for permeable fracture zones in geothermal areas. Ward [1972] suggested that microearthquakes might be used to locate active faults that may channel hot fluids toward the surface.

Brune and Allen [1967] and Thatcher and Brune [1971] have reported microearthquake activity associated with geothermal areas in the Imperial Valley south of the Salton Sea. Ward and Bjornsson [1971] located over 2100 earthquakes in Iceland, and they noted a difference between the continuous microearthquake activity in the geothermal areas and the larger shocks followed by aftershock sequences in other parts of the island. Lange and Westphal [1969] detected microearthquake activity near The Geysers, California, during a recording period of 5 days. In several other geothermal areas of the western United States they detected such activity in recording periods of similar duration. Hamilton and Muffer [1972] located 53 microearthquakes within $10 \mathrm{~km}$ of the then producing area at The Geysers, California, in a 3-week period.

Microearthquakes may have other uses in geothermal exploration. Hamilton and Muffler [1972] suggested that the maximum focal depths of microearthquakes may be a temperature indicator. Brace and Byerlee [1970] showed that high temperature may prevent stick slip (i.e., earthquakes) and induce stable sliding.

The Long Valley caldera is an elliptical depression 19 by 29 $\mathrm{km}$ (Figure 1). Rhyolitic extrusion has occurred in the last $1500 \mathrm{yr}$, and hot spring activity persists to the present time. Bailey et al. [1976] have noted a difference in the character of faulting inside and outside the caldera, and they suggest that the material inside the caldera was decoupled for a time from the uplift of the Sierras to the south and west.

Gumper and Scholz [1971] and Ryall et al. [1972] located microearthquakes in Chalfant Valley and in a zone trending northwestward from Bishop toward Mammoth. Their net-

\footnotetext{
${ }^{1}$ Now at Kansas Geological Survey, University of Kansas, Lawrence, Kansas 66044.

Copyright $\odot 1976$ by the American Geophysical Union.
}

works suffered from azimuthal control and distance with respect to Long Valley, and events smaller than magnitude 1.5 in Long Valley could not be detected. Pitt and Steeples [1975] detected only two microearthquakes in the Long Valley caldera while they confirmed the seismicity pattern established by the earlier investigators south and east of Long Valley. There has been considerable historic seismicity in the region, including the great 1872 earthquake in Owens Valley, about $100 \mathrm{~km}$ southeast of the Long Valley caldera. Pitt and Steeples [1975] interpreted microearthquake focal mechanisms just outside the southeastern edge of the caldera in terms of right lateral strike slip. Those microearthquakes were located near the epicenters of two magnitude 6.0 earthquakes that occurred within the last $50 \mathrm{yr}$ [California Department of Water Resources, 1964].

\section{THE NeTwORK}

The instruments used for this study were the USGS portable seismograph systems that have been described in detail by Eaton et al. [1970]. Sixteen of these systems were operated at 20 different locations during the recording period. The array was centered on the eastern half of the caldera, and six locations from a previous study by Pitt and Steeples [1975] were reoccupied (Table 1).

\section{Crustal Model}

A shallow crustal model for the immediate Long Valley area was described by Hill [1976]. The microearthquakes recorded (discussed later) were mostly outside the caldera, so the local Hill model was bypassed in favor of a regional model derived from the reversed refraction profile between Mono Lake and China Lake, California, described by Eaton [1966]. This profile passes near the center of the seismic array of the present study. The model consists of the following sequence of horizontal constant velocity layers, where velocity is in kilometers per second and depth is in kilometers:

\begin{tabular}{cc}
$P$ Wave Velocity & Depth to Top of Layer \\
\cline { 2 - 2 } 3.0 & 0.0 \\
6.0 & 1.7 \\
6.4 & 15.0 \\
6.9 & 28.0 \\
7.9 & 54.0
\end{tabular}

Using this model, well-recorded blasts within the network were located to within $1 \mathrm{~km}$ horizontally and $2 \mathrm{~km}$ vertically. It is assumed that well-recorded earthquakes that occurred within the network were located to this same order of accuracy and that earthquake locations outside the network may be in 


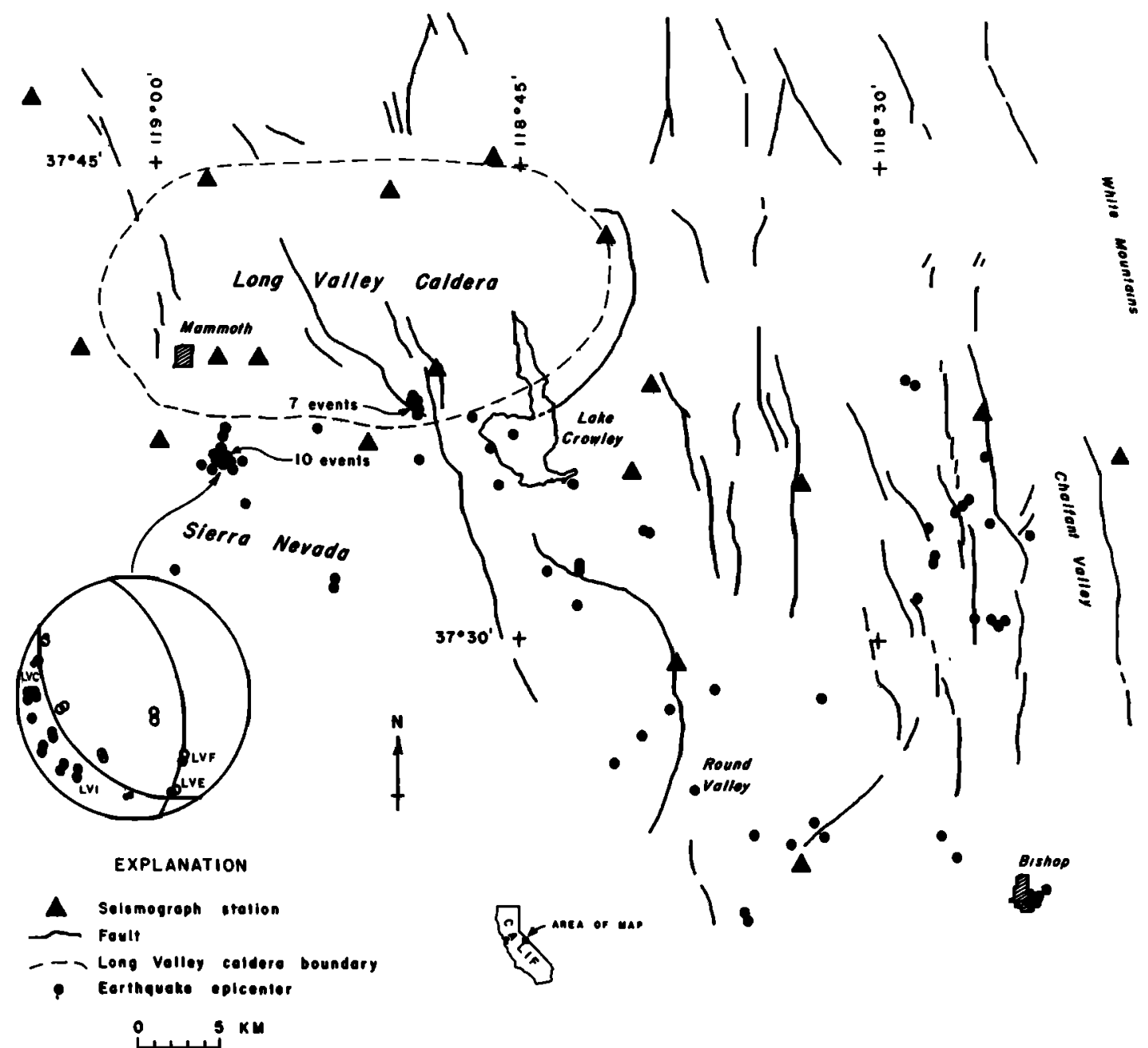

Fig. 1. Microearthquakes in the vicinity of Long Valley caldera from April 27 to June 2, 1973. On the nodal plane solution, solid circles represent initial compression, and open circles initial dilatation; smaller circles indicate less reliable first motions. Stations mentioned in Figure 2 are designated by three letters on the nodal plane solution.

error by $2 \mathrm{~km}$ or more. Relative locations of closely spaced events within the network may be accurate to a few hundred meters. No elevation or station corrections were used.

\section{Selection of Data SeT}

All local events that appeared at three or more stations were analyzed. $P$ arrivals were picked and timed to $0.01 \mathrm{~s}$ by using station WWVB or internal chronometer corrected to WWVB. $S$ arrivals were timed on the five three-component stations and where possible on the 11 one-component stations. Reading errors (as distinguished from picking errors) are no more than $\pm 0.02 \mathrm{~s}$.

$S$ arrivals were only used to locate events when the $P$-only solution produced $S$ residuals suggesting poor quality locations. A Wadati diagram was used to establish the proper $V p / V s$ ratio for the region (1.70). The uncertainties in picking the $S$ arrivals may be $\frac{1}{2} \mathrm{~s}$ or more, but balanced $S$ residuals (i.e., some positive, some negative) are considered to indicate stable (good quality) hypocenter solutions. Events with fewer than five $P$ arrivals picked to better than $0.25 \mathrm{~s}$ were dropped from the data set unless the location was within $5 \mathrm{~km}$ of the edge of the Long Valley caldera, in which case all events with three or more stations were kept.

Hypocenters and magnitudes from the signal duration [Lee et al., 1972] were determined by Geiger's method using the computer program HYPO71 [Lee and Lahr, 1972] (Table 2). The magnitudes determined here may differ from the classical Richter local magnitudes by as much as one third of a magnitude unit and should therefore be regarded as local magnitude approximations.

\section{Microearthquake Distribution}

The events listed in Table 2 are plotted in Figure 1 (except for two events outside the map area). The activity is concentrated in a WNW trending band extending from near Bishop almost to Mammoth. Focal depths range from 1 to 15 km with no systematic pattern to the depth distribution. The seismicity pattern is very similar to that found by Gumper and Scholz [1971] and Ryall et al. [1972]. It is also similar to the pattern found by Pitt and Steeples [1975], except that the swarm near $37^{\circ} 36^{\prime} \mathrm{N}, 118^{\circ} 57^{\prime} \mathrm{W}$ (Figure 1) is in an area in which only two events were detected in a 20 -day recording period in 1970.

The 1973 data alone (Figure 1) are insufficient to present the trends that emerge when all our data are plotted together. In Figure 3 all of the microearthquake data from 1970 and 1973 are displayed, along with station locations from 1973. Approximately 50 of these events (of over 200 plotted) had fewer than five readings, and perhaps a half dozen (shallow events within $15 \mathrm{~km}$ of the D in Sierra Nevada (Figure 3 )) of the 50 could be 
TABLE 1. Seismograph Station Data

\begin{tabular}{|c|c|c|c|c|c|}
\hline Location & Station & North Latitude & West Longitude & Elevation, m & Instruments \\
\hline 1 & LVA & $37^{\circ} 36.16^{\prime}$ & $118^{\circ} 59.68^{\prime}$ & 2768 & USGS Portable \\
\hline 2 & LVB & $37^{\circ} 36.13^{\prime}$ & $118^{\circ} 51.15^{\prime}$ & 2310 & USGS Portable \\
\hline 3 & LVC & $37^{\circ} 34.97^{\prime}$ & $118^{\circ} 33.17^{\prime}$ & 2231 & USGS Portable \\
\hline 4 & LVD & $37^{\circ} 45.14^{\prime}$ & $118^{\circ} 46.03^{\prime}$ & 2195 & USGS Portable \\
\hline 5 & LVE & $37^{\circ} 46.82^{\prime}$ & $119^{\circ} 04.96^{\prime}$ & 2365 & USGS Portable \\
\hline 6 & LVF & $37^{\circ} 39.03^{\prime}$ & $119^{\circ} 03.01^{\prime}$ & 2755 & USGS Portable \\
\hline 7 & LVG & $37^{\circ} 44.41^{\prime}$ & $118^{\circ} 57.81^{\prime}$ & 2292 & USGS Portable \\
\hline 8 & LVH & $37^{\circ} 38.83^{\prime}$ & $118^{\circ} 57.33^{\prime}$ & 2365 & USGS Portable \\
\hline 9 & LVI & $37^{\circ} 44.20^{\prime}$ & $118^{\circ} 50.36^{\prime}$ & 2121 & USGS Portable \\
\hline 10 & LVJ & $37^{\circ} 38.50^{\prime}$ & $118^{\circ} 48.37^{\prime}$ & 2140 & USGS Portable \\
\hline 11 & LVK & $37^{\circ} 42.73^{\prime}$ & $118^{\circ} 41.28^{\prime}$ & 2249 & USGS Portable \\
\hline 12 & LVL & $37^{\circ} 38.05^{\prime}$ & $118^{\circ} 39.38^{\prime}$ & 2316 & USGS Portable \\
\hline 13 & LVM & $37^{\circ} 35.22^{\prime}$ & $118^{\circ} 40.42^{\prime}$ & 2170 & USGS Portable \\
\hline 14 & LVN & $37^{\circ} 35.87^{\prime}$ & $118^{\circ} 20.25^{\prime}$ & 1792 & USGS Portable \\
\hline 15 & LVO & $37^{\circ} 29.21^{\prime}$ & $118^{\circ} 38.19^{\prime}$ & 1719 & USGS Portable \\
\hline 16 & LVP & $37^{\circ} 23.03^{\prime}$ & $118^{\circ} 33.14^{\prime}$ & 1451 & USGS Portable \\
\hline 17 & LVR & $37^{\circ} 38.82^{\prime}$ & $118^{\circ} 55.67^{\prime}$ & 2341 & USGS Portable \\
\hline 18 & LVS & $37^{\circ} 35.47^{\prime}$ & $118^{\circ} 50.07^{\prime}$ & 2426 & USGS Portable \\
\hline 19 & LVT & $37^{\circ} 55.86^{\prime}$ & $118^{\circ} 56.30^{\prime}$ & 2077 & USGS Portable \\
\hline 20 & LVU & $37^{\circ} 36.71^{\prime}$ & $118^{\circ} 23.92^{\prime}$ & 1378 & USGS Portable \\
\hline 21 & DFOD & $37^{\circ} 33.18^{\prime}$ & $117^{\circ} 45.16^{\prime}$ & 2173 & $\begin{array}{l}\text { USGS Telemetered } \\
\text { NCER }\end{array}$ \\
\hline 22 & TIN & $37^{\circ} 03.30^{\prime}$ & $118^{\circ} 13.70^{\prime}$ & 1195 & Cal Tech \\
\hline
\end{tabular}

explosions. The hypocenters of these poorly located events could be in error by more than $5 \mathrm{~km}$ horizontally and $10 \mathrm{~km}$ vertically. The good events from 1970 are plotted in the work by Pitt and Steeples [1975].

A north-south trend is apparent in Chalfant Valley. A larger and more extensive trend from northern Owens Valley toward Long Valley caldera reinforces the trend suggested in Figure 1, including the abrupt decrease in activity at the edge of Long Valley caldera. It is particularly noteworthy that few events were detected within the Long Valley caldera. This is discussed in detail later.

\section{Nodal Plane Solution}

The directions of first motion of $P$ arrivals were noted when sufficiently strong and classified on three quality levels: (1) certainly correct because of the amplitude and character of the arrival; (2) probably correct because of the character of the arrival but of lower amplitude than events in the first class; and (3) less certain than classes (1) and (2) but still worth using in order to increase the density of coverage on the focal sphere. Computer program HYPO7I provides an equal-area projection of first motions on the lower hemisphere.

Figure 1 shows a nodal plane solution with an arrow pointing to the epicenter on the map. The solution, a composite of two nearly identical events, has nodal planes that can be drawn by using arrivals from both events but using only two fortuitously located stations. The two events used had hypocenters within a few hundred meters of each other. At 13 stations (of 15 operating) the seismograms could almost be traced one event upon the other. Figure $2 a$ shows typical examples of this similarity. The remaining two stations (LVE and LVF) possessed nodal character (much smaller amplitude) for the $P$ arrival and showed a reversal of sense of first motion from one event to the other (Figure $2 b$ ). It is known [e.g., Helmberger, 1974] that $P$ nodes and $S$ nodes coincide at only two diametrically opposite points on a focal sphere and also that the $P$ nodal planes are mutually perpendicular, intersecting along a straight line connecting the two points. This can be visualized by imagining a perfect vertical strip-slip focal sphere viewed from above. The north pole of the sphere is a point of intersection of the two $P$ nodal planes. The north pole is also a point of intersecting $S$ nodes. The south pole of the focal sphere is the only other point where both $P$ nodes and $S$ nodes coincide. This special case of perfect vertical strike slip can be generalized to the present (or any other) case simply by rotating the focal sphere.

Figure $2 c$ shows that station LVE was at or very near a point of coincidence of $\boldsymbol{P}$ node and $\boldsymbol{S}$ node, so that the diametrically opposed nodal points are both known to within a very few degrees. In addition, station LVF showed $P$ nodal character, so that three $\boldsymbol{P}$ nodal points are known and thus one $\boldsymbol{P}$ nodal plane is uniquely determined. The second $\boldsymbol{P}$ nodal plane is also uniquely determined, since it must pass through the two diametrically opposed $\boldsymbol{P}$ and $\boldsymbol{S}$ node coincidence points and at the same time be perpendicular to the $\boldsymbol{P}$ nodal plane previously determined.

The nodal planes drawn by this method are in total agreement with the first motions for both events at the other 13 stations as shown in Figure 1. In this case the data agree well with the double-couple mechanism theory.

This particular pair of events shows how important radiation pattern can be in determining the amplitude of $S$ waves. When this severe attenuation of $S$ waves was first observed by the authors, the tendency was to think it might be related to attenuation by magma as in studies by Kubota and Berg [1967] and Matumoto [1971] at Mt. Katmai, Alaska, and Pitt [1974] at Yellowstone. This is an example that indicates that the radiation pattern can drastically affect amplitudes but that near total attentuation is limited to small areas of the focal sphere.

As originally conceived, part of this investigation was to include attenuation data from local earthquakes that sent energy through the Long Valley caldera in a study similar to that at Yellowstone by Pitt [1974]. This study produced negative results, as the ray paths involved did not penetrate to sufficient depth in the caldera to encounter significant low-velocity material, other than that in the upper 3 or $4 \mathrm{~km}$ described in the refraction study of $H$ ill [1976]. The ray paths in the caldera were too shallow to detect the low-velocity material described by Steeples and Iyer [1976] and Steeples [1975]. 
TABLE 2. Microearthquakes in the Long Valley Vicinity From April 27 to May 30, 1973

\begin{tabular}{|c|c|c|c|c|c|c|c|c|c|c|c|c|}
\hline \multirow[b]{2}{*}{ Date } & \multirow[b]{2}{*}{ Origin } & \multicolumn{2}{|c|}{ Epicenter } & \multirow{2}{*}{$\begin{array}{c}\text { Depth } \\
\text { of } \\
\text { Focus, } \\
\text { km }\end{array}$} & \multirow[b]{2}{*}{$M$} & \multirow[b]{2}{*}{$N$} & & & & & & \\
\hline & & $\begin{array}{c}\text { North } \\
\text { Latitude }\end{array}$ & $\begin{array}{c}\text { West } \\
\text { Longitude }\end{array}$ & & & & $\begin{array}{l}\text { Gap, } \\
\text { deg }\end{array}$ & $\underset{\mathrm{km}}{D_{\mathrm{min}}}$ & $\begin{array}{c}\mathbf{r m s} \text {, } \\
\mathrm{s}\end{array}$ & $\begin{array}{c}E R H, \\
\mathrm{~km}\end{array}$ & $\begin{array}{c}E R Z, \\
\mathrm{~km}\end{array}$ & \\
\hline April 27 & $13 \mathrm{~h} 05 \mathrm{~m} 09.13 \mathrm{~s}$ & $37^{\circ} 37.01^{\prime}$ & $118^{\circ} 46.90^{\prime}$ & 5.00 & 1.29 & 3 & 187 & 6.5 & 0.00 & & & $\mathrm{AD}$ \\
\hline April 30 & $23 \mathrm{~h} 05 \mathrm{~m} 04.48 \mathrm{~s}$ & $37^{\circ} 27.72^{\prime}$ & $118^{\circ} 38.63^{\prime}$ & 5.36 & 1.79 & 7 & 163 & 2.8 & 0.08 & 1.1 & 1.4 & BC \\
\hline April 30 & $07 \mathrm{~h} 28 \mathrm{~m} \mathrm{43.08s}$ & $37^{\circ} 33.35^{\prime}$ & $118^{\circ} 39.68^{\prime}$ & 7.99 & 1.34 & 5 & 143 & 3.6 & 0.06 & 1.0 & 2.0 & BD \\
\hline May 1 & $05 \mathrm{~h} 58 \mathrm{~m} 15.53 \mathrm{~s}$ & $37^{\circ} 36.56^{\prime}$ & $118^{\circ} 57.06^{\prime}$ & 8.35 & 2.07 & 23 & 172 & 4.2 & 0.14 & 0.5 & 0.8 & $\mathrm{AC}$ \\
\hline May 1 & $07 \mathrm{~h} 35 \mathrm{~m} \mathrm{29.05s}$ & $37^{\circ} 30.49^{\prime}$ & $118^{\circ} 25.09^{\prime}$ & 9.87 & 2.44 & 16 & 269 & 18.2 & 0.15 & 1.6 & 3.0 & BD \\
\hline May 1 & $07 \mathrm{~h} 40 \mathrm{~m} 38.65 \mathrm{~s}$ & $37^{\circ} 30.61^{\prime}$ & $118^{\circ} 24.91^{\prime}$ & 13.01 & 2.59 & 10 & 270 & 18.5 & 0.12 & 3.4 & 3.7 & CD \\
\hline May 1 & $08 \mathrm{~h} \mathrm{59m} \mathrm{58.56s}$ & $37^{\circ} 28.37^{\prime}$ & $118^{\circ} 36.80^{\prime}$ & 5.00 & 1.59 & 3 & 163 & 2.6 & 0.03 & & & \\
\hline May 1 & $10 \mathrm{~h} 37 \mathrm{~m} \mathrm{34.68s}$ & $37^{\circ} 35.40^{\prime}$ & $118^{\circ} 57.43^{\prime}$ & 8.06 & 1.89 & 11 & 187 & 6.3 & 0.10 & 0.8 & 1.3 & $\mathrm{AD}$ \\
\hline May 1 & $11 \mathrm{~h} 27 \mathrm{~m} 25.07 \mathrm{~s}$ & $37^{\circ} 35.41^{\prime}$ & $118^{\circ} 57.43^{\prime}$ & 7.70 & 1.42 & 10 & 197 & 6.3 & 0.12 & 1.2 & 1.9 & BD \\
\hline May 1 & $19 \mathrm{~h} 14 \mathrm{~m} 18.85 \mathrm{~s}$ & $37^{\circ} 35.17^{\prime}$ & $118^{\circ} 57.65^{\prime}$ & 5.00 & 1.44 & 6 & 201 & 10.6 & 0.13 & 2.1 & 12.0 & \\
\hline May 1 & $22 \mathrm{~h} 43 \mathrm{~m} \mathrm{24.89s}$ & $37^{\circ} 35.22^{\prime}$ & $118^{\circ} 56.84^{\prime}$ & 5.00 & 1.66 & 7 & 181 & 4.5 & 0.11 & 1.3 & 2.6 & BD \\
\hline May 2 & $03 \mathrm{~h} 14 \mathrm{~m} 21.92 \mathrm{~s}$ & $37^{\circ} 30.71^{\prime}$ & $118^{\circ} 26.14^{\prime}$ & 9.53 & 1.67 & 10 & 264 & 13.0 & 0.12 & 2.7 & 2.7 & CD \\
\hline May 2 & $09 \mathrm{~h} 40 \mathrm{~m} 58.78 \mathrm{~s}$ & $37^{\circ} 33.34^{\prime}$ & $118^{\circ} 39.62^{\prime}$ & 9.56 & 1.07 & 8 & 115 & 7.9 & 0.23 & 2.1 & 4.6 & BB \\
\hline May 2 & $10 \mathrm{~h} 37 \mathrm{~m} 08.42 \mathrm{~s}$ & $37^{\circ} 35.75^{\prime}$ & $118^{\circ} 25.63^{\prime}$ & 7.88 & 2.08 & 12 & 265 & 11.2 & 0.15 & 3.3 & 2.9 & CD \\
\hline May 2 & $11 \mathrm{~h} 58 \mathrm{~m} \mathrm{33.73s}$ & $37^{\circ} 36.26^{\prime}$ & $118^{\circ} 57.14^{\prime}$ & 7.40 & 1.15 & 16 & 152 & 3.7 & 0.13 & 0.7 & 0.9 & \\
\hline May 2 & $14 \mathrm{~h} 21 \mathrm{~m} \mathrm{17.69s}$ & $37^{\circ} 34.09^{\prime}$ & $118^{\circ} 56.27^{\prime}$ & 5.00 & 1.18 & 4 & 234 & 6.3 & 0.04 & & & \\
\hline May 2 & $17 \mathrm{~h} 06 \mathrm{~m} \mathrm{59.16s}$ & $37^{\circ} 35.47^{\prime}$ & $118^{\circ} 56.36^{\prime}$ & 2.00 & 1.35 & 4 & 193 & 5.1 & 0.08 & & & \\
\hline May 2 & $17 \mathrm{~h} 53 \mathrm{~m} 44.03 \mathrm{~s}$ & $37^{\circ} 35.88^{\prime}$ & $118^{\circ} 46.14^{\prime}$ & 9.61 & 1.51 & 8 & 135 & 8.5 & 0.14 & 1.4 & 4.3 & \\
\hline May 3 & $09 \mathrm{~h} 11 \mathrm{~m} \mathrm{58.34 \textrm {s }}$ & $37^{\circ} 33.30^{\prime}$ & $118^{\circ} 23.73^{\prime}$ & 12.62 & 1.21 & 6 & 285 & 14,2 & 0.13 & 5.5 & 3.9 & D \\
\hline May 3 & $14 \mathrm{~h} 36 \mathrm{~m} \mathrm{53.39s}$ & $37^{\circ} 32.07^{\prime}$ & $118^{\circ} 43.74^{\prime}$ & 13.69 & 0.95 & 14 & 217 & 7.6 & 0.37 & 2.3 & 2.7 & C \\
\hline May 3 & $15 \mathrm{~h} 03 \mathrm{~m} \mathrm{24.08s}$ & $37^{\circ} 26.88^{\prime}$ & $118^{\circ} 39.77^{\prime}$ & 7.20 & 1.23 & 4 & 174 & 4.9 & 0.00 & & & \\
\hline May 5 & $09 \mathrm{~h} 21 \mathrm{~m} \mathrm{51.38s}$ & $37^{\circ} 25.99^{\prime}$ & $118^{\circ} 40.95^{\prime}$ & 6.49 & 1.01 & 9 & 189 & 7.2 & 0.05 & 0.5 & 0.9 & \\
\hline May 5 & $09 \mathrm{~h} 40 \mathrm{~m} \mathrm{06.85s}$ & $37^{\circ} 30.66^{\prime}$ & $118^{\circ} 25.34^{\prime}$ & 9.57 & 1.71 & 13 & 181 & 12.2 & 0.14 & 1.4 & 3.0 & \\
\hline May 8 & $09 \mathrm{~h} 09 \mathrm{~m} 04.61 \mathrm{~s}$ & $37^{\circ} 35.59^{\prime}$ & $118^{\circ} 56.85^{\prime}$ & 4.89 & 0.86 & 7 & 171 & 4.3 & 0.04 & 0.6 & 1.4 & \\
\hline May 8 & $09 \mathrm{~h} 10 \mathrm{~m} 28.41 \mathrm{~s}$ & $37^{\circ} 35.86^{\prime}$ & $118^{\circ} 57.19^{\prime}$ & 6.86 & 1.10 & 9 & 165 & 3.7 & 0.06 & 0.5 & 0.8 & \\
\hline May 9 & $06 \mathrm{~h} 41 \mathrm{~m} 14.48 \mathrm{~s}$ & $37^{\circ} 23.13^{\prime}$ & $118^{\circ} 26.74^{\prime}$ & 9.04 & 1.78 & 25 & 247 & 9.4 & 0.24 & 1.3 & 1.6 & \\
\hline May 9 & $08 \mathrm{~h} 06 \mathrm{~m} 43.82 \mathrm{~s}$ & $37^{\circ} 23.78^{\prime}$ & $118^{\circ} 27.43^{\prime}$ & 9.09 & 1.36 & 15 & 235 & 8.5 & 0.17 & 1.1 & 1.3 & \\
\hline May 9 & $08 \mathrm{~h} 58 \mathrm{~m} 44.41 \mathrm{~s}$ & $37^{\circ} 36.57^{\prime}$ & $118^{\circ} 53.31^{\prime}$ & 2.96 & 0.83 & 7 & 161 & 3.3 & 0.04 & 0.4 & 1.2 & \\
\hline May 9 & $16 \mathrm{~h} 04 \mathrm{~m} 56.41 \mathrm{~s}$ & $37^{\circ} 31.83^{\prime}$ & $118^{\circ} 52.54^{\prime}$ & 5.54 & 1.02 & 9 & 204 & 8.2 & 0.08 & 0.8 & 2.1 & \\
\hline May 9 & $16 \mathrm{~h} 47 \mathrm{~m} \mathrm{59.12 \textrm {s }}$ & $37^{\circ} 31.57^{\prime}$ & $118^{\circ} 52.63^{\prime}$ & 3.33 & 1.28 & 12 & 208 & 8.7 & 0.07 & 0.6 & 3.8 & \\
\hline May 9 & $19 \mathrm{~h} 17 \mathrm{~m} \mathrm{23.33s}$ & $37^{\circ} 35.70^{\prime}$ & $118^{\circ} 57.57^{\prime}$ & 9.08 & 2.49 & 17 & 162 & 3.2 & 0.09 & 0.5 & 0.6 & \\
\hline May 9 & $20 \mathrm{~h} 32 \mathrm{~m} \mathrm{59.19s}$ & $37^{\circ} 35.46^{\prime}$ & $118^{\circ} 57.22^{\prime}$ & 6.25 & 1.74 & 11 & 177 & 3.8 & 0.09 & 0.8 & 1.2 & \\
\hline May 9 & $21 \mathrm{~h} 23 \mathrm{~m} 59.54 \mathrm{~s}$ & $37^{\circ} 35.47^{\prime}$ & $118^{\circ} 56.97^{\prime}$ & 6.85 & 1.34 & 10 & 175 & 4.2 & 0.08 & 0.8 & 1.2 & \\
\hline May 9 & $22 \mathrm{~h} 28 \mathrm{~m} 40.76 \mathrm{~s}$ & $37^{\circ} 20.91^{\prime}$ & $118^{\circ} 35.43^{\prime}$ & 12.42 & 2.52 & 14 & 235 & 5.2 & 0.09 & 1.0 & 0.6 & \\
\hline May 10 & $05 \mathrm{~h} 28 \mathrm{~m} \mathrm{28.62s}$ & $37^{\circ} 21.16^{\prime}$ & $118^{\circ} 35.53^{\prime}$ & 11.83 & 1.78 & 15 & 235 & 4.9 & 0.11 & 0.9 & 0.6 & \\
\hline May 10 & $16 \mathrm{~h} 48 \mathrm{~m} 24.17 \mathrm{~s}$ & $37^{\circ} 34.79^{\prime}$ & $118^{\circ} 45.82^{\prime}$ & 6.34 & 1.37 & 13 & 138 & 7.8 & 0.08 & 0.4 & 1.1 & \\
\hline May 10 & $21 \mathrm{~h} 08 \mathrm{~m} 17.75 \mathrm{~s}$ & $37^{\circ} 35.57^{\prime}$ & $118^{\circ} 56.94^{\prime}$ & 6.56 & 1.34 & 9 & 172 & 4.2 & 0.08 & 0.9 & 1.3 & \\
\hline May 11 & $08 \mathrm{~h} 14 \mathrm{~m} \mathrm{03.58s}$ & $37^{\circ} 35.54^{\prime}$ & $118^{\circ} 56.85^{\prime}$ & 5.00 & 1.05 & 6 & 203 & 4.3 & 0.06 & 1.0 & 1.8 & \\
\hline May 12 & $04 \mathrm{~h} 48 \mathrm{~m} 10.85 \mathrm{~s}$ & $37^{\circ} 35.56^{\prime}$ & $118^{\circ} 49.10^{\prime}$ & 5.81 & 1.02 & 9 & 148 & 3.2 & 0.09 & 0.8 & 1.2 & \\
\hline May 12 & $12 \mathrm{~h} 23 \mathrm{~m} \mathrm{44.34s}$ & $37^{\circ} 34.05^{\prime}$ & $118^{\circ} 26.87^{\prime}$ & 0.92 & 1.74 & 12 & 144 & 9.4 & 0.17 & 1.5 & 142.1 & \\
\hline May 12 & $12 \mathrm{~h} 28 \mathrm{~m} \mathrm{26.15s}$ & $37^{\circ} 34.24^{\prime}$ & $118^{\circ} 26.58^{\prime}$ & 4.03 & 1.80 & 11 & 146 & 9.8 & 0.15 & 1.7 & 6.0 & \\
\hline May 13 & $02 \mathrm{~h} 31 \mathrm{~m} 50.50 \mathrm{~s}$ & $37^{\circ} 35.35^{\prime}$ & $118^{\circ} 58.01^{\prime}$ & 9.27 & 2.56 & 19 & 164 & 2.9 & 0.15 & 0.8 & 0.8 & \\
\hline May 13 & $04 \mathrm{~h} 35 \mathrm{~m} \mathrm{36.23s}$ & $37^{\circ} 36.50^{\prime}$ & $118^{\circ} 45.24^{\prime}$ & 10.35 & 0.85 & 10 & 157 & 5.9 & 0.11 & 1.3 & 2.1 & \\
\hline May 13 & $05 \mathrm{~h} 42 \mathrm{~m} 36.22 \mathrm{~s}$ & $37^{\circ} 35.90^{\prime}$ & $118^{\circ} 57.25^{\prime}$ & 7.73 & 1.63 & 12 & 164 & 3.6 & 0.08 & 0.7 & 1.0 & \\
\hline May 13 & $09 \mathrm{~h} 50 \mathrm{~m} 34.42 \mathrm{~s}$ & $37^{\circ} 35.36^{\prime}$ & $118^{\circ} 56.99^{\prime}$ & 6.41 & 0.97 & 8 & 199 & 4.2 & 0.06 & 0.8 & 1.1 & \\
\hline May 13 & $17 \mathrm{~h} 48 \mathrm{~m} 45.60 \mathrm{~s}$ & $37^{\circ} 31.30^{\prime}$ & $118^{\circ} 28.44^{\prime}$ & 9.43 & 1.29 & 8 & 149 & 9.7 & 0.15 & 1.7 & 3.3 & \\
\hline May 14 & $10 \mathrm{~h} 22 \mathrm{~m} 56.58 \mathrm{~s}$ & $37^{\circ} 32.65^{\prime}$ & $118^{\circ} 27.72^{\prime}$ & 5.76 & 2.00 & 16 & 143 & 9.1 & 0.13 & 0.8 & 2.5 & \\
\hline May 17 & $08 \mathrm{~h} 12 \mathrm{~m} \mathrm{14.19s}$ & $37^{\circ} 22.27^{\prime}$ & $118^{\circ} 23.02^{\prime}$ & 6.49 & 1.71 & 14 & 266 & 15.0 & 0.12 & 2.6 & 4.5 & \\
\hline May 17 & $08 \mathrm{~h} 43 \mathrm{~m} 34.11 \mathrm{~s}$ & $37^{\circ} 21.99^{\prime}$ & $118^{\circ} 23.36^{\prime}$ & 9.27 & 2.97 & 20 & 120 & 14.6 & 0.16 & 0.8 & 1.4 & \\
\hline May 17 & $17 \mathrm{~h} 44 \mathrm{~m} 19.26 \mathrm{~s}$ & $37^{\circ} 21.77^{\prime}$ & $118^{\circ} 23.73^{\prime}$ & 5.00 & 1.22 & 6 & 309 & 14.1 & 0.13 & 5.5 & 5.1 & \\
\hline May 18 & $14 \mathrm{~h} 54 \mathrm{~m} 39.74 \mathrm{~s}$ & $37^{\circ} 34.45^{\prime}$ & $118^{\circ} 26.31^{\prime}$ & 2.40 & 1.08 & 5 & 167 & 9.3 & 0.06 & 1.4 & 21.9 & \\
\hline May 19 & $11 \mathrm{~h} 53 \mathrm{~m} 09.82 \mathrm{~s}$ & $37^{\circ} 32.20^{\prime}$ & $118^{\circ} 42.43^{\prime}$ & 12.85 & 0.99 & 22 & 145 & 6.3 & 0.13 & 0.5 & 0.7 & \\
\hline May 19 & $11 \mathrm{~h} 53 \mathrm{~m} 40.73 \mathrm{~s}$ & $37^{\circ} 32.20^{\prime}$ & $118^{\circ} 42.45^{\prime}$ & 12.22 & 1.12 & 23 & 145 & 6.3 & 0.11 & 0.4 & 0.6 & \\
\hline May 20 & $15 \mathrm{~h} 18 \mathrm{~m} 15.67 \mathrm{~s}$ & $37^{\circ} 23.55^{\prime}$ & $118^{\circ} 33.78^{\prime}$ & 11.93 & 1.55 & 24 & 225 & 21.1 & 0.39 & 3.3 & 6.5 & \\
\hline May 20 & $15 \mathrm{~h} 24 \mathrm{~m} 05.75 \mathrm{~s}$ & $37^{\circ} 37.41^{\prime}$ & $118^{\circ} 49.46^{\prime}$ & 9.09 & 1.28 & 13 & 119 & 2.6 & 0.09 & 0.6 & 0.9 & \\
\hline May 20 & $16 \mathrm{~h} 14 \mathrm{~m} 36.17 \mathrm{~s}$ & $37^{\circ} 37.55^{\prime}$ & $118^{\circ} 49.50^{\prime}$ & 9.26 & 1.03 & 11 & 115 & 2.4 & 0.09 & 0.7 & 1.2 & \\
\hline May 20 & $2 \mathrm{lh} 03 \mathrm{~m} \mathrm{54.33s}$ & $37^{\circ} 37.52^{\prime}$ & $118^{\circ} 49.28^{\prime}$ & 10.73 & 1.11 & 7 & 126 & 2.3 & 0.07 & 0.8 & 1.1 & \\
\hline May 21 & $06 \mathrm{~h} 29 \mathrm{~m} 28.56 \mathrm{~s}$ & $37^{\circ} 37.47^{\prime}$ & $118^{\circ} 49.42^{\prime}$ & 11.26 & 0.44 & 5 & 140 & 2.5 & 0.04 & 2.3 & 1.6 & \\
\hline May 21 & $08 \mathrm{~h} 21 \mathrm{~m} \mathrm{20.18s}$ & $37^{\circ} 37.35^{\prime}$ & $118^{\circ} 49.36^{\prime}$ & 12.40 & 0.37 & 7 & 144 & 2.6 & 0.04 & 0.6 & 1.0 & \\
\hline May 21 & $1 \mathrm{lh} 12 \mathrm{~m} 41.04 \mathrm{~s}$ & $37^{\circ} 37.58^{\prime}$ & $118^{\circ} 49.39^{\prime}$ & 11.51 & 0.57 & 11 & 116 & 2.3 & 0.11 & 0.9 & 1.9 & \\
\hline May 21 & $11 \mathrm{~h} 13 \mathrm{~m} 39.21 \mathrm{~s}$ & $37^{\circ} 37.05^{\prime}$ & $118^{\circ} 49.20^{\prime}$ & 10.86 & 0.44 & 10 & 134 & 2.9 & 0.12 & 1.0 & 2.1 & \\
\hline May 21 & $13 \mathrm{~h} 03 \mathrm{~m} \mathrm{09.61 \textrm {s }}$ & $37^{\circ} 57.88^{\prime}$ & $118^{\circ} 42.58^{\prime}$ & 14.50 & 1.29 & 17 & 273 & 20.4 & 0.14 & 1.1 & 1.8 & \\
\hline May 21 & $20 \mathrm{~h} 36 \mathrm{~m} 56.25 \mathrm{~s}$ & $37^{\circ} 31.03^{\prime}$ & $118^{\circ} 42.52^{\prime}$ & 0.32 & 2.87 & 18 & 221 & 8.4 & 0.21 & 1.2 & 0.9 & B \\
\hline May 21 & $22 \mathrm{~h} 58 \mathrm{~m} 15.89 \mathrm{~s}$ & $37^{\circ} 32.48^{\prime}$ & $118^{\circ} 27.76^{\prime}$ & 9.73 & 1.81 & 8 & 218 & 9.2 & 0.17 & 3.6 & 3.8 & \\
\hline May 22 & $06 \mathrm{~h} 21 \mathrm{~m} 16.33 \mathrm{~s}$ & $37^{\circ} 38.17^{\prime}$ & $118^{\circ} 28.96^{\prime}$ & 11.30 & 1.55 & 14 & 159 & 7.9 & 0.14 & 1.4 & 2.2 & \\
\hline May 22 & $10 \mathrm{~h} 11 \mathrm{~m} 22.46 \mathrm{~s}$ & $37^{\circ} 32.09$ & $118^{\circ} 59.20^{\prime}$ & 1.93 & 0.85 & 5 & 267 & 7.6 & 0.08 & 2.3 & 20.8 & \\
\hline May 23 & $06 \mathrm{~h} 04 \mathrm{~m} 14.24 \mathrm{~s}$ & $37^{\circ} 33.48^{\prime}$ & $118^{\circ} 27.95^{\prime}$ & 7.94 & 1.23 & 6 & 207 & 8.2 & 0.11 & 4.4 & 3.3 & \\
\hline May 23 & $07 \mathrm{~h} 05 \mathrm{~m} 48.23 \mathrm{~s}$ & $37^{\circ} 24.23^{\prime}$ & $118^{\circ} 32.69^{\prime}$ & 5.00 & 1.61 & 13 & 259 & 19.9 & 0.14 & 2.9 & 14.6 & \\
\hline May 23 & $09 \mathrm{~h} 00 \mathrm{~m} 57.80 \mathrm{~s}$ & $37^{\circ} 37.10^{\prime}$ & $118^{\circ} 26.06^{\prime}$ & 2.27 & 1.98 & 15 & 197 & 34.1 & 0.08 & 0.8 & 94.7 & $\mathrm{CI}$ \\
\hline May 23 & $17 \mathrm{~h} 22 \mathrm{~m} \mathrm{35.34s}$ & $37^{\circ} 23.72^{\prime}$ & $118^{\circ} 32.28^{\prime}$ & 5.00 & 1.93 & 8 & 261 & 20.8 & 0.12 & 3.4 & 19.6 & \\
\hline May 25 & $07 \mathrm{~h} 48 \mathrm{~m} 36.20 \mathrm{~s}$ & $37^{\circ} 34.83^{\prime}$ & $118^{\circ} 42.68^{\prime}$ & 8.17 & 0.55 & 5 & 310 & 3.4 & 0.03 & 1.0 & 0.4 & \\
\hline
\end{tabular}


TABLE 2. (continued)

\begin{tabular}{|c|c|c|c|c|c|c|c|c|c|c|c|c|}
\hline \multirow[b]{2}{*}{ Date } & \multirow[b]{2}{*}{ Origin } & \multicolumn{2}{|c|}{ Epicenter } & \multirow{2}{*}{$\begin{array}{c}\text { Depth } \\
\text { of } \\
\text { Focus, } \\
\text { km }\end{array}$} & \multirow[b]{2}{*}{$M$} & \multirow[b]{2}{*}{$N$} & \multirow[b]{2}{*}{$\begin{array}{l}\text { Gap, } \\
\text { deg }\end{array}$} & \multirow[b]{2}{*}{$\begin{array}{c}D_{\mathrm{min}} \\
\mathrm{km}\end{array}$} & \multirow[b]{2}{*}{$\begin{array}{c}\mathrm{rms} \\
\mathrm{s}\end{array}$} & \multirow[b]{2}{*}{$\underset{\mathrm{km}}{E R H}$} & \multirow[b]{2}{*}{$\begin{array}{c}E R Z, \\
\mathrm{~km}\end{array}$} & \multirow[b]{2}{*}{$Q$} \\
\hline & & $\begin{array}{c}\text { North } \\
\text { Latitude }\end{array}$ & $\begin{array}{c}\text { West } \\
\text { Longitude }\end{array}$ & & & & & & & & & \\
\hline $\begin{array}{l}\text { May } 28 \\
\text { May } 28\end{array}$ & $\begin{array}{l}08 \mathrm{~h} 13 \mathrm{~m} 31.43 \mathrm{~s} \\
14 \mathrm{~h} 43 \mathrm{~m} 16.81 \mathrm{~s}\end{array}$ & $\begin{array}{l}37^{\circ} 23.78^{\prime} \\
37^{\circ} 28.09^{\prime}\end{array}$ & $\begin{array}{l}118^{\circ} 35.20^{\prime} \\
118^{\circ} 32.47^{\prime}\end{array}$ & $\begin{array}{l}9.71 \\
8.58\end{array}$ & $\begin{array}{l}1.58 \\
2.00\end{array}$ & $\begin{array}{r}9 \\
13\end{array}$ & $\begin{array}{l}268 \\
239\end{array}$ & $\begin{array}{l}20.9 \\
12.8\end{array}$ & $\begin{array}{l}0.07 \\
0.21\end{array}$ & $\begin{array}{l}3.2 \\
3.0\end{array}$ & $\begin{array}{l}6.3 \\
5.2\end{array}$ & $\begin{array}{l}C D \\
C D\end{array}$ \\
\hline $\begin{array}{l}\text { May } 28 \\
\text { May } 29\end{array}$ & $\begin{array}{l}15 \mathrm{~h} 24 \mathrm{~m} 19.72 \mathrm{~s} \\
13 \mathrm{~h} 04 \mathrm{~m} 33.77 \mathrm{~s}\end{array}$ & $\begin{array}{l}37^{\circ} 25.13^{\prime} \\
37^{\circ} 38.04^{\prime}\end{array}$ & $\begin{array}{l}118^{\circ} 37.55^{\prime} \\
118^{\circ} 28.48^{\prime}\end{array}$ & $\begin{array}{l}8.84 \\
8.20\end{array}$ & $\begin{array}{l}1.52 \\
1.99\end{array}$ & $\begin{array}{r}9 \\
14\end{array}$ & $\begin{array}{l}282 \\
171\end{array}$ & $\begin{array}{r}19.1 \\
7.1\end{array}$ & $\begin{array}{l}0.08 \\
0.16\end{array}$ & $\begin{array}{l}2.8 \\
1.6\end{array}$ & $\begin{array}{l}4.5 \\
2.9\end{array}$ & $\begin{array}{l}\mathrm{CD} \\
\mathrm{BC}\end{array}$ \\
\hline May 30 & $12 \mathrm{~h} 25 \mathrm{~m} 19.55 \mathrm{~s}$ & $37^{\circ} 33.65^{\prime}$ & $118^{\circ} 25.40^{\prime}$ & 7.09 & 2.81 & 16 & 184 & 6.1 & 0.15 & 1.4 & 1.7 & BD \\
\hline
\end{tabular}

$M$ is the magnitude of the earthquake; $N$ is the number of stations used in locating the earthquake; the gap is the largest azimuthal separation between stations; $D_{\min }$ is the epicentral distance to the nearest station; rms is the root mean square error of the time residuals (rms $=\Sigma_{i} R_{i}^{2} / N$, where $R_{t}$ is the observed seismic wave arrival time less the computed time at the $i$ th station); $E R H$ is the standard error of the epicenter $\left(E R H=S D X^{2}+S D Y^{2}\right.$, where $S D X$ and $S D Y$ are the standard errors in latitude and longitude, respectively, of the epicenter); $E R Z$ is the standard error of the depth (left blank if it is $\geq 10$ ).

$Q$ is the solution quality of the hypocenter (given as two letters) based on both the statistical measure of the solution and the nature of the station distribution with respect to the earthquake. Each of these two factors is rated independently according to the following scheme. For the statistical measure (first letter), for A, rms $<0.15, E R H \leq 1.0$, and $E R Z \leq 2.0$. For $\mathrm{B}$, rms $<0.30, E R H \leq 2.5$, and $E R Z \leq 5.0$. For $\mathrm{C}, \mathrm{rms}<0.50$, and $E R H \leq 5.0$. For $\mathrm{D}$, the values are other than those given above. For the station distribution (second letter), for $\mathrm{A}, N \geq 6$, the gap $\leq 90$, and $D_{\operatorname{mln}} \leq$ depth or 5 . For $\mathrm{B}, N \geq 6$, the gap $\leq 135$, and $D_{\min } \leq 2$ times depth or 10 . For $\mathrm{C}, N \geq 6$, the gap $\leq 180$, and $D_{\min } \leq 50$. For $\mathrm{D}$, the values are other than those given above.

\section{Discussion}

Pitt and Steeples [1975] noted the similarity between their 1970 seismicity and the historic seismicity from 1934 to 1970 , as well as the similarity of their seismicity to patterns deter- mined by Gumper and Scholz [1971] and Ryall et al. [1972]. The pattern of seismicity in Figure 3 is very similar to the historic seismicity. It is probably not valid to try to correlate this short sample of epicenters to specific faults in the Long Valley vicinity. There are faults both inside and outside the a

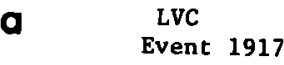
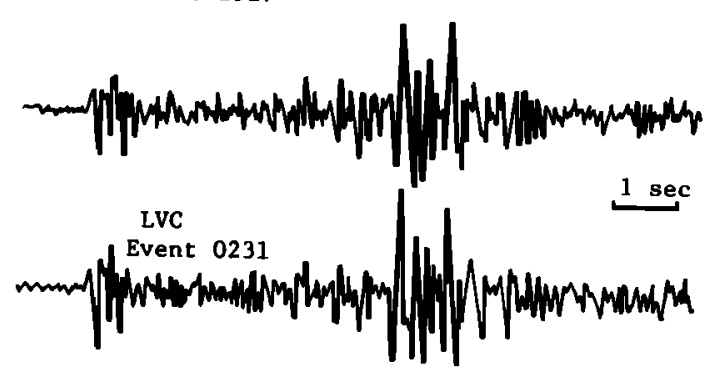

VI

Event 1917

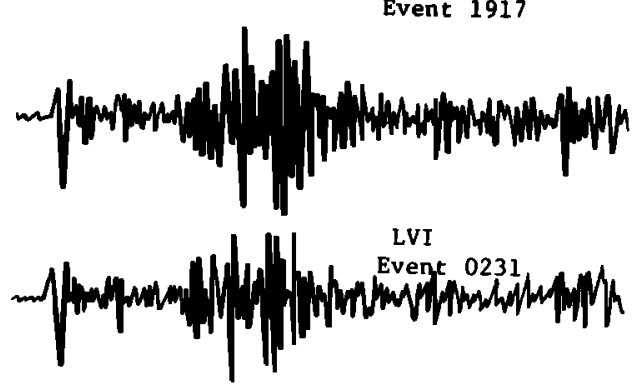

b

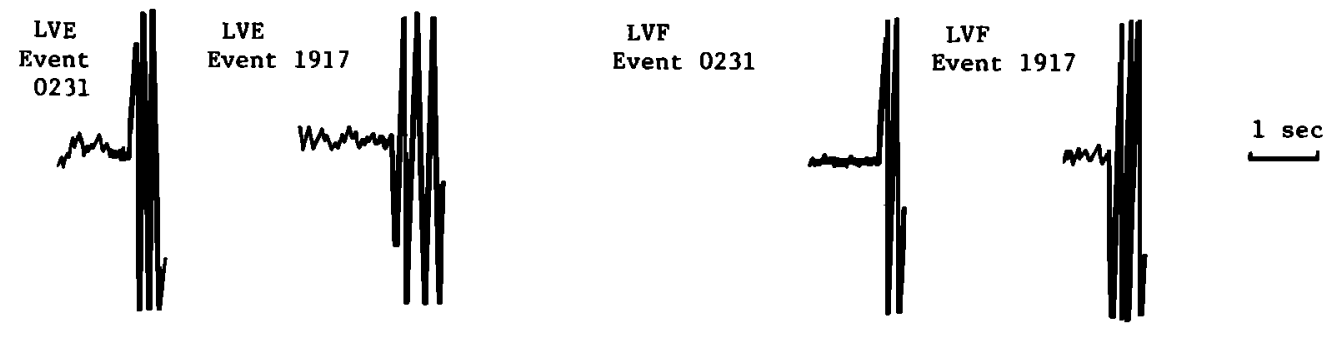

C

LVE
Event 1917

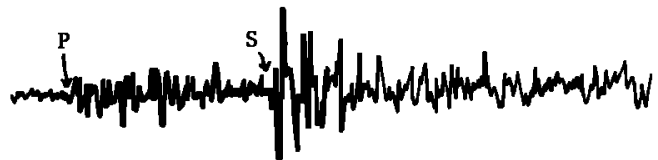

$1 \mathrm{sec}$

LVE

Event 0231

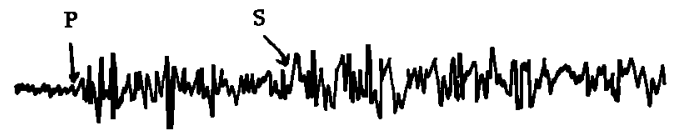

Fig. 2. Seismograms used to construct nodal plane solution near Mammoth in Figure 1. (a) Low-gain trace seismograms from station LVC (left) and LVI (right) showing similarity, which was typical at all but two stations between events. (b) High-gain trace seismograms from stations LVE (left) and LVF (right) showing reversal of first motion from one event to the other. The low-gain $P$ arrivals were emergent at these two stations relative to the low-gain arrivals at the other 13 stations. Compare low-gain arrivals in $(a)$ with low-gain $P$ arrivals in $(c)$. (c) The low-gain trace seismograms from station LVE showing a weak $P$ arrival for both events (compare with $(b)$ ). Note almost total disappearance of $S$ arrival for the 0231 event at station LVE. 


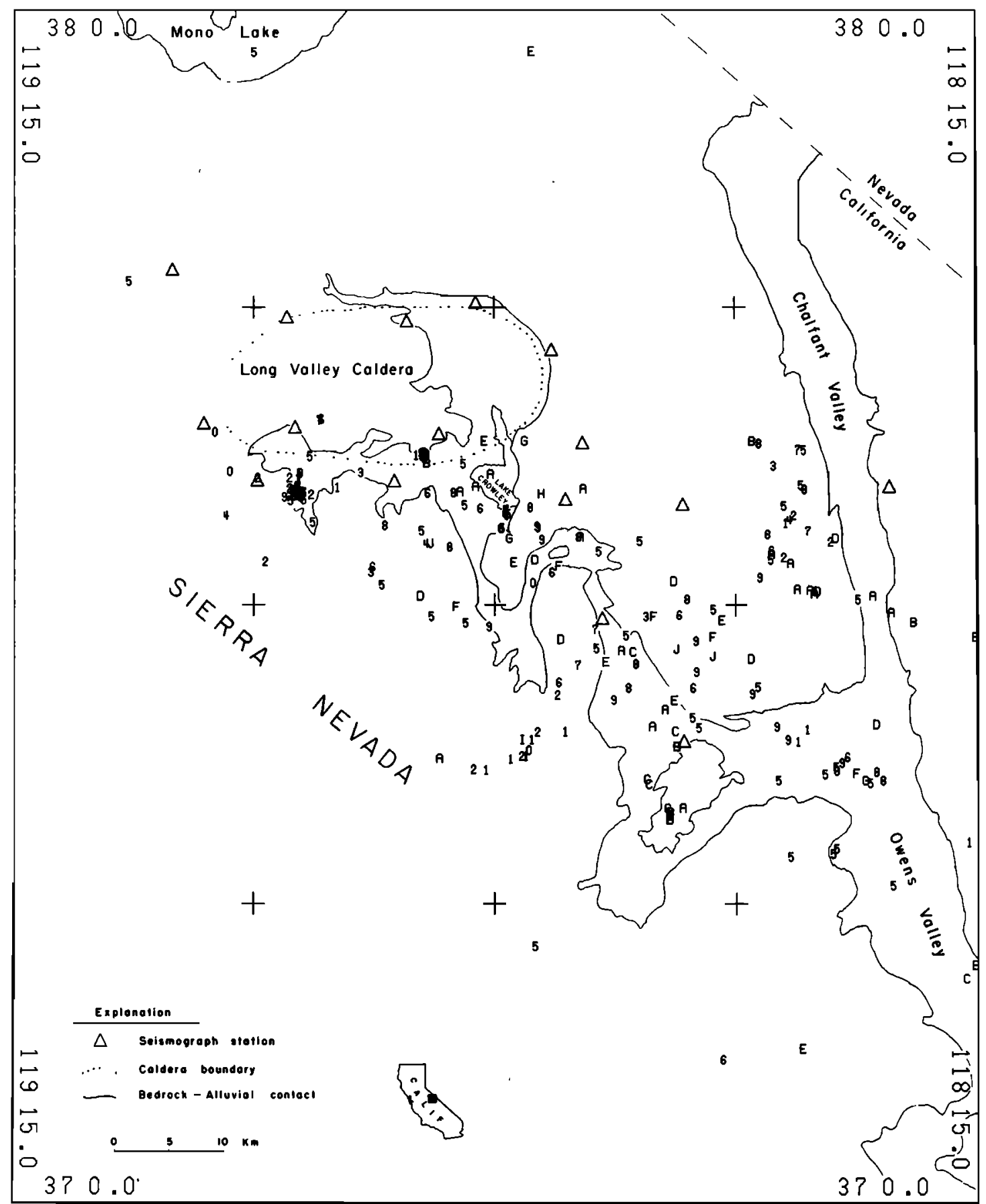

Fig. 3. All microearthquakes from both studies (1970 and 1973). Triangles show 1973 station locations; 1970 stations are not plotted but are shown in the work by Pitt and Steeples [1975]. Note the low level of activity in the Long Valley caldera. Focal depths of microearthquakes in kilometers are indicated by numbers and letters $(A=10 \mathrm{~km}, B=11 \mathrm{~km}$, etc. $)$. Note the north-south trend from Chalfant Valley to Owens Valley and the northwest-southeast trend from Long Valley caldera to Owens Valley.

caldera that show recent displacements [see Bailey et al., 1976].

Several nodal plane solutions presented in the work by Pitt and Steeples [1975] indicate a relative tension axis oriented within a few degrees of east-west. The normal dip-slip solution shown in Figure 1 also has a tension axis oriented roughly east-west, a pressure axis that is roughly vertical. The east-west extension in the Basin and $R$ ange province is well known, so the relative tension axis is in agreement with tectonic deformation to the east of the Sierra Nevada.

Ward [1972] has pointed out that microearthquakes occur in most major geothermal areas. He also stated,
The observation that large numbers of microearthquakes are often found within but not outside of geothermal areas during short periods of field recording suggests that seismic activity within the geothermal areas may be a far more continuous process than seismic activity in most other areas, even though all the seismic activity may be along the same fault system and in response to the same regional stresses.

The data presented here from Long Valley are opposite to the above observation; i.e., there are many microearthquakes just outside the Long Valley caldera but few within the caldera where geothermal prospects are thought to be best. There are several possible explanations: 
1. Either Long Valley is not a major geothermal area, or it is an exception to Ward's [1972] observation.

2. The recording period coincided with an aseismic period in Long Valley. Pitt and Steeples [1975] located two microearthquakes in west central Long Valley from a 20-day recording period in 1970 , and activity outside the caldera was comparable to the present study.

3. The microearthquakes in Long Valley were too small to be detected by our network. The network was capable of detecting events down to about magnitude zero or perhaps -0.5 .

4. Some part of the upper crust in Long Valley is not strong enough to support the stresses required for detectable earthquakes. This might be true if magma or partial melt were present in the upper $15 \mathrm{~km}$ of the crust. Such a soft zone could effectively isolate the upper $15 \mathrm{~km}$ from regional stresses that are obviously present as shown by microearthquake activity near the Long Valley caldera.

5. High temperatures could produce stable sliding instead of brittle fracture [Brace and Byerlee, 1970] in the upper crust in the caldera.

6. Gumper and Scholz [1971] noted a similar lack of activity in the vicinity of the Long Valley caldera and northern Chalfant Valley. As shown in Figure 3, seismic activity virtually ceases along an east-west line projected along the southern edge of the caldera. This may have some tectonic significance that is not yet understood but is not thought to be a result of inadequate station coverage. Gumper and Scholz [1971] suggested that the Owens Valley is a spreading ridge. This would explain the presence of volcanic activity in the Long Valley vicinity but might not explain the petrology of the more silicic igneous rocks.

Whatever the explanation, the only microearthquakes recorded within the caldera are at the extreme southeastern edge of the caldera, and they are about $10 \mathrm{~km}$ deep. These may be on the Hylton Creek fault (the only fault in Figure 1 that crosses the southern caldera boundary), and they are probably associated with regional deformation. It is impossible to tell from this small cluster of events if a zone facilitating upward movement of hot water or steam is present. The termination of seismic activity at the southern edge of the caldera may suggest permeable zones (or at least fractured material) close to that edge.

Although microearthquakes have been of some use in locating apparent fracture zones at some of the earlier mentioned geothermal localities, the method is far from proven in the more general sense of geothermal exploration. The difficulty of locating fracture zones with microearthquakes is exemplified by the San Andreas fault. One merely has to look at a National Center for Earthquake Research seismicity report for central California [e.g., Wesson et al., 1974] to see that the seismicity is typically placed a kilometer or two off the fault trace. If one is interested in drilling for a geothermal resource, the exploration technique should be capable of locating fracture zones to better than of the order of a couple of kilometers. Another limitation of microearthquakes is that their absence does not imply the absence of active faults. Some parts of the San Andreas fault have broken the surface in historic time but yet have little or no microearthquake activity at the present time, as can be seen in the previously mentioned report of Wesson et al.

Acknowledgments. The field work for this investigation was performed by John Coakley and Tim Hitchcock. Tapes were played back and records prepared by Jeanne Taylor. Publication was authorized by the Director, U.S. Geological Survey.

\section{REFERENCES}

Bailey, R. A., G. B. Dalrymple, and M. A. Lanphere, Volcanism, structure, and geochronology of Long Valley caldera, Mono County, California, J. Geophys. Res., 81, this issue, 1976.

Brace, W. F., and J. D. Byerlee, California earthquakes: Why only shallow focus?, Science, 168, 1573, 1970.

Brune, J. N., and C. R. Allen, A microearthquake survey of the San Andreas fault system in southern California, Bull. Seismol. Soc. Amer. 57, 277, 1967.

California Department of Water Resources, Crustal strain and fault movement investigation-faults and earthquake epicenters in California, Calif. Div. Mines Geol. Bull., 116-2. 1964.

Eaton, J. P., Crustal structure in northern and central California from seismic evidence, Calif. Div. Mines Geol. Bull., 190, 419-426, 1966.

Eaton, J. P., M. E. O'Neill, and J. N. Murdock, Aftershocks of the 1966 Parkfield-Cholame, California earthquake: A detailed study, Bull. Seismol. Soc. Amer., 60, 1151-1198, 1970.

Facca, G., and F. Tonani, Theory and technology of a geothermal field, Bull. Volcanol. 27(2), 143-189, 1964.

Gumper, F. J., and C. H. Scholz, Microseismicity and tectonics of the Nevada seismic zone, Bull. Seismol. Soc. Amer., 61, 1413-1432, 1971.

Hamilton, R. M., and L. J. P. Muffler, Microearthquakes at The Geysers geothermal area, California, J. Geophys. Res., 77. 2081-2086, 1972.

Helmberger, D. V., Generalized ray theory for shear dislocations, Bull. Seismol. Soc. Amer., 64, 45-64, 1974.

Hill, D. P., Structure of Long Valley caldera, California, from a seismic refraction experiment, J. Geophys. Res., 81, this issue, 1976.

Kubota, S., and E. Berg, Evidence for magma in the Katmai volcanic range, Bull. Volcanol., 31, 175-214, 1967.

Lange, A. L., and W. H. Westphal, Microearthquakes near The Geysers, Sonoma County, California, J. Geophys. Res., 74, 4377, 1969.

Lee, W. H. K., and J. C. Lahr, HYPO71: A computer program for determining hypocenter, magnitude, and first motion pattern of local earthquakes, open file report, 100 pp., U.S. Geol. Surv., Menlo Park, Calif.. 1972.

Lee, W. H., R. E. Bennett, and K. L. Meagher, A method of estimating magnitude of local earthquakes from signal duration, open file report, 28 pp., U.S. Geol. Surv., Menlo Park, Calif., 1972.

Matumoto, T., Seismic body waves observed in the vicinity of Mount Katmai, Alaska, and evidence for the existence of molten chambers, Geol. Soc. Amer. Bull., 82, 2905-2920, 1971.

Pitt, A. M., Evidence from local earthquakes for the existence of a region of seismic body wave attenuation in the upper crust under the Yellowstone caldera (abstract), Eos Trans. AGU, 55, 1191, 1974.

Pitt, A. M., and D. W. Steeples, Microearthquakes in the Mono Lake-northern Owens Valley region from September 28 to October 18, 1970, Bull. Seismol. Soc. Amer., 65, 835-844, 1975.

Ryall, A.S., W. U. Savage, and D. B. Slemmons, Seismic potential in the western Basin and Range/eastern Sierra Nevada region, Nevada and California (abstract), EOS Trans. AGU, 53, 442, 1972.

Steeples, D. W., Teleseismic $P$-delays in geothermal exploration with application to Long Yalley, California, Ph.D. thesis, Stanford Univ., Stanford, Calif., 1975

Steeples, D. W., and H. M. Iyer, Low-velocity zone under Long Valley as determined from teleseismic events, J. Geophys. Res., 81, this issue, 1976

Thatcher, W., and J. N. Brune, Seismic study of an oceanic ridge earthquake swarm in the Gulf of California, Geophys. J. Roy. Astron. Soc., 22, 473, 1971.

Ward, P. L., Microearthquakes: Prospecting tool and possible hazard in the development of geothermal resources, Geothermics, 1, 3-12, 1972.

Ward, P. L., and S. Bjornsson, Microearthquakes, swarms, and the geothermal areas of Iceland, J. Geophys. Res., 76, 3953-3982, 1971.

Wesson, R. L., F. W. Lester, and K. L. Meagher, Catalog of earthquakes along the San Andreas fault system in central California, January-March 1973, open file report, 47 pp., U.S. Geol. Surv., Menlo Park, Calif., 1974.

(Received December 26, 1974; revised July 22, 1975 accepted July 24,1975 .) 